\title{
Nanoparticles: breakthrough in COVID-19 prevention, diagnosis and treatment
}

\section{Type}

Review paper (After Acceptance of EiC)

\section{Keywords}

nanoparticles, COVID-19, SARS-CoV-2, coronavirus disease 2019

\begin{abstract}
Coronavirus disease 2019 (COVID-19) is an infectious disease caused by severe acute respiratory syndrome coronavirus 2 (SARS-CoV-2), declared a global pandemic by the World Health Organization (WHO). The three key principles in management of the COVID-19 pandemic are prevention, early detection and targeted treatment. Vaccine-based prevention together with early detection have already proven their efficacy in controlling the pandemic, early detection of the infected patients could substantially accelerate the implementation of treatment, but also help to identify the infection hotspots, whereas the targeted treatment might destroy the virus and minimize damage to healthy tissue. Nanoparticles hold great promise with respect to these above-mentioned aspects. They may also be the solution to the emerging clinical problems, such as: reinfection, pregnant-related COVID-19 and coinfection. Here, we aim to discuss the potential applications of nanoparticles to combat the COVID-19 pandemic.
\end{abstract}




\section{REVIEW}

\section{Nanoparticles: a breakthrough in COVID-19 prevention, diagnosis and treatment}

Aleksandra Skwarek ${ }^{1}$, Aleksandra Gąsecka ${ }^{1}$, Miłosz J. Jaguszewski², Łukasz Szarpak $^{3,4}$, Tomasz Dzieciątkowski ${ }^{5}$, Krzysztof J. Filipiak ${ }^{1}$

${ }^{1} 1^{\text {st }}$ Chair and Department of Cardiology, Medical University of Warsaw, Warsaw, Poland

${ }^{2} 1^{\text {st }}$ Department of Cardiology, Medical University of Gdańsk, Gdańsk, Poland

${ }^{3}$ Maria Skłodowska-Curie Medical Academy in Warsaw, Warsaw, Poland

${ }^{4}$ Maria Skłodowska-Curie Białystok Oncology Center, Białystok, Poland

${ }^{5}$ Chair and Department of Medical Microbiology, Medical University of Warsaw, Warsaw, Poland

\section{Corresponding author:}

Aleksandra Gąsecka

$1^{\text {st }}$ Chair and Department of Cardiology

Medical University of Warsaw, Poland

Email: aleksandra.gasecka@wum.edu.pl

Phone: (048) 225991951 


\begin{abstract}
Coronavirus disease 2019 (COVID-19) is an infectious disease caused by severe acute respiratory syndrome coronavirus 2 (SARS-CoV-2), declared a global pandemic by the World Health Organization (WHO). The three key principles in management of the COVID-19 pandemic are prevention, early detection and targeted treatment. Vaccine-based prevention together with early detection have already proven their efficacy in controlling the pandemic, early detection of the infected patients could substantially accelerate the implementation of treatment, but also help to identify the infection hotspots, whereas the targeted treatment might destroy the virus and minimize damage to healthy tissue. Nanoparticles hold great promise with respect to these above-mentioned aspects. They may also be the solution to the emerging clinical problems, such as: reinfection, pregnant-related COVID-19 and coinfection. Here, we aim to discuss the potential applications of nanoparticles to combat the COVID-19 pandemic.
\end{abstract} Keywords: coronavirus disease 2019, COVID-19, SARS-CoV-2, nanoparticles 


\section{Introduction}

Coronavirus disease 2019 (COVID-19) is an infectious disease caused by severe acute respiratory syndrome coronavirus 2 (SARS-CoV-2), first reported in Wuhan, China in late 2019. Compared to previously identified coronaviruses, COVID-19 is associated with lower mortality, but easier transmission. So far, COVID-19 has reached 220 countries, infected more than 203,991,344 people, and caused 4,313,922 deaths (www.worldometers.info/coronavirus; August 10). The pandemic poses a global risk, and requires highly effective control measures (1). The three key principles in management of the COVID-19 pandemic are (i) prevention, (ii) early detection and (iii) targeted treatment (2). Vaccine-based prevention has already been proven to be an effective way to stop infectious diseases and is currently indispensable in controlling the pandemic. Early detection of infected patients is crucial to identify the infection hotspots and accelerate treatment. Finally, targeted treatment might destroy the virus while minimizing damage to healthy tissue. Hence, novel technologies to facilitate prevention, early detection and targeted treatment of COVID-19 are of paramount importance for global health. Many recent technological developments are based on nanoparticles.

Nanoparticles, which are defined as particles of any shape with a diameter between 1 and $100 \mathrm{~nm}$ (3), hold great promise for the prevention, detection, and treatment of COVID-19, from vaccine development via nanoparticle-based point-of-care diagnostic tests to the antiviral mechanism of action of several potential therapeutic agents. Recently, many excellent reviews have been published discussing the potential applications of nanoparticles to combat the COVID-19 pandemic (4-8). However, previous reviews focus on nanoparticles from a technological and/or industrial perspective. Here, we aim to discuss the role of nanoparticles in prevention, early detection and treatment of COVID-19 from a clinical perspective. In addition, 
we have illustrated each of the discussed topics with clear figures to facilitate better understanding of the topic.

\section{Prevention: Vaccine development and nano-filters}

The genetic sequence of SARS-CoV-2 was published on January 11, 2020, triggering intense global activity to develop a vaccine against the disease (9). The first COVID-19 vaccine candidate entered human clinical testing as early as March 16, 2020. Currently, there are four vaccines authorized by the European Medicines Agency for use in the European Union: mRNAbased Comirnaty ${ }^{\circledR}$ (Pfizer/BioN-Tech, US/Germany) and Moderna ${ }^{\circledR}$ (Moderna, Spain), and vector-based Vaxzevria ${ }^{\circledR}$ (AstraZeneca, Sweden) and Janssen ${ }^{\circledR}($ Janssen-Cilag International, Belgium). So far, $31.4 \%$ of the world population have received at least one dose of a COVID19 vaccine (https://ourworldindata.org/covid-vaccinations, August 16).

mRNA vaccines have been developed as an alternative to live-attenuated vaccines and subunit vaccines, and combine their advantages: maintaining potency in activating both cellular and humoral immunity without the risks of reversion to a pathogenic form that might cause disease $(10,11)$. However, even the most potent vaccine candidate will not exert the required action without an efficient delivery system. The main challenge faced by mRNA vaccines for clinical approval is their intracellular delivery (12-14). Because of mRNA sensitivity to degradation by ribonucleases, mRNA is highly unstable under physiological conditions. Several strategies have been developed for RNA delivery, including RNA-conjugates, viral vectors, microparticles and nanoparticles. While linking RNA to molecules offers some level of protection against degradation, it promotes binding to serum proteins and subsequent aggregation that can lead to vascular blockage (13). Viral vectors, in turn, have several limitations, including immunogenicity, potential carcinogenesis, broad tropism and production difficulties (14). 
Lipid nanoparticles are particularly promising delivery systems, since they (i) can be synthesized with relative ease in a scalable manner, (ii) protect the mRNA against degradation, (iii) can be targeted to the desired cell type by surface decoration with ligands, and if needed, (iv) can be co-delivered with adjuvants, (v) exhibit great potential for mucosal vaccinations (12, 15). This technology is proven safe, as it has been used in medicine for years, for example, for the administration of some antibiotics with high toxicity. The working principle of an mRNAbased vaccine delivered by lipid nanoparticles is presented in Figure 1.

Currently, five mRNA-based vaccines utilizing a nanoparticle platform are undergoing clinical trials, including the Comirnaty ${ }^{\circledR}$ and Moderna ${ }^{\circledR}$ vaccines (3). There is also growing interest in protein subunit vaccines supported by nanoparticle-based delivery systems, with five clinical trials already investigating their effectiveness (3). Nanoparticles' beneficial effects vary depending on the type of the vaccine (13).

Protein subunit vaccines, which are generally poorly immunogenic and often fail to mobilize efficient immune responses, clearly benefit from immunopotentiating properties of nanoparticles. The presence of an immunostimulating agent is indispensable for this type of nanoparticles $(13,16)$. mRNA vaccines are believed to combine the advantages of subunit vaccines and live-attenuated vaccines, by exhibiting a highly efficient induction of the immune response and no risk of reverting into an active virus (13). However, they are highly unstable under physiological conditions, as previously described. Consequently, they benefit to the greatest extent from the delivery by nanoparticles. Altogether, nanoparticle-based delivery systems are the key to vaccine delivery.

Except for lipid nanoparticles, there are several other nanoparticles, which may find application in vaccine delivery, including (i) exosomes, (ii) virus-like particles (VLP) and (iii) polymeric nanoparticles (17). The advantages and disadvantages of these nanoparticles are discussed below. 
Exosomes are naturally occuring nanoparticles released by numerous cells types to body fluids and have multiple biological functions, including intercellular communication (18). Their biological origin, as opposed to the synthetic origin of other nanoparticles (e.g. polymeric nanoparticles), is the key to their functionality (18). The advantages of exosomes include their similarity to cell membranes and resistance to recognition and clearance by the immune system. Exosomes express markers derived from the parent cell on their surface, which allow to target and bind to specific cell types. This specificity limits the scope of cells they can interact with and the number of clinical scenarios, in which they can be used. The specific targeting can be also designed individually and achieved by the means of genetic engineering (19). Recently, mesenchymal stem cell (MSC)-derived exosomes were reported to have properties ideal for the potential applications in vaccination and treatment strategies against COVID-19. MSC-derived exosomes are (i) abundantly released by MSCs, (ii) highly stable in vivo, (iii) feasible for storage, (iv) resistant to immune-mediated pre-term degradation and (v) able to cross the bloodbrain barrier, which makes them worth considering for delivery of vaccine against SARS-CoV2 (20). However, production of MSC-derived exosomes is a complex procedure which requires well-controlled conditions. Therefore, exosomes are not yet used in clinical applications (21).

VLP are assemblages of viral proteins, shaped as an envelope, that are able to trigger immune response through activation of pathogen-associated molecular-pattern recognition receptors (13). VLP do not contain genetic information and consequently remain non-replicant and non-infectious. As a result, VLP elicit a strong immune response against the viral antigen, but can be produced easily in a scalable manner. However, the potential applications of VLP in vaccine development are hampered by difficulties in protein assembly and structure formation, possibility of excessive immunostimulation and poor loading capacity during the production process (22). 
Polymeric nanoparticles have gained much attention for their effectiveness and biocompatibility in vaccine delivery. Their advantages include protection from degradation of the carried molecule, sustained release and intrinsic adjuvant activity. At the moment, there is no sufficient data regarding mRNA vaccination with polymeric nanoparticles. However, they have been proven effective in delivery of Hepatitis B virus antigen (23), live Newcastle disease virus (24) and DNA encoding epitopes of Mycobacterium tuberculosis (25) during vaccination.

Apart from the vaccination, effective prevention also includes the implementation of high-quality personal protective equipment. A polybenzimidazole nano-filter has been recently developed, which, due to its unique mechanical, thermal and chemical durability, maintains filtering efficiency, even after a cleaning procedure (26). This reusable nano-filtered face mask could help to meet the challenges arising from the supply shortage of face masks. One significant advantage of the use of nanoparticle-based filters is their potential for direct antiviral activity, as shown with the ReSpimask ${ }^{\circledR}$ VK (RESPILON), a mask already available on the market, which was shown to prevent the spread of COVID-19 (27).

\section{Detection: Nanosensors}

Detecting virus particles is challenging, not only because of their extremely small size, but also because of their unique physical and chemical characteristics. Nanosensors are becoming a favorable diagnostic tool, showing great ability to detect SARS-CoV-2 infection at a very early stage and thus warning clinicians before overt symptoms develop in patients with low viral loads.

Lateral flow antigen detection for SARS-CoV-2 is a point-of-care approach, which is already being used for diagnosing COVID-19. Special attention is paid to the highly conserved $\mathrm{N}$ (nucleocapsid) protein, as its expression reaches high values during infection. In commercially available lateral flow immunochromatographic assays, also called immunostrips, 
a paper-like membrane strip is coated with two lines, containing anti-N-protein antibody and capture antibodies, respectively. The patient's sample is deposited in the sample pad and flows across the strip by capillary action. In the conjugation pad, the N-protein contained in the patient's nasopharyngeal secretion binds to the anti-N-protein antibody labeled with a gold nanoparticle. As the sample passes the test line, the conjugate binds to the immobilized anti-Nprotein antibody. At the control line, the conjugate of rabbit IgG antibody labeled with a gold nanoparticle is immobilized by the capture antibodies, serving as a control.

The application of nanoparticles (e.g. colloidal gold) in lateral flow assay has significantly improved its clinical sensitivity and specificity with some of the commercialized rapid tests, showing sensitivity of $97-99 \%$ (28). The working principle of this assay is shown in Figure 2.

\section{Treatment: nanoparticle-based drugs and delivery systems}

Currently, there are over 35 European Medicines Agency (EMA) or Food and Drug Administration (FDA) approved nanoparticle-based medicines and over 55 other nanoparticle technologies that are being evaluated in ongoing clinical trials (29). The main types of nanoparticles currently used in clinics include lipid nanoparticles and polymeric nanoparticles $(4,7,30)$. The advantages of lipid nanoparticles include their biocompatibility, biodegradability and non-toxicity. In addition, the solubility or stability of lipid nanoparticles can be modified to further enhance the efficacy of nanoparticle-associated drugs (30). Polymeric nanoparticles, in turn, have low biocompatibility compared to lipid nanoparticles, but can be tailored to various sizes in order to reach a specific target and allow for stable drug release $(4,7)$. Therefore, both lipid and polymeric nanoparticles are likely to enter clinical practice for the treatment of COVID-19. 
The potential use of nanoparticles to treat viral infections, including COVID-19, holds great promise for (i) nanoparticle-based delivery systems for both drugs and siRNA, (ii) direct antiviral activity and (iii) stimulation of immune system activity.

The AP2-associated protein kinase 1 (AAK1) has emerged as a molecular target in the treatment of SARS-CoV-2 infection. AAK1 is a pivotal regulator of endocytosis, thus its inhibition would hinder viral entry into the target cells. When used in high dosages, substances like sunitinib and erlotinib act as AAK1 inhibitors, consequently blocking viral entry (30). The combination of sunitinib and erlotinib has already proven to effectively suppress systemic infection in a study with mice (30). A significant drawback of AAK1-based therapy is, however, its high toxicity. This could be avoided by coupling the AAK1 inhibitors with nanoparticles, leading to optimized distribution to the target location and an increased safety profile.

Another potential method to treat COVID-19 would be the use of small interfering RNA (siRNA). siRNA silences the viral mRNA and thus blocks its gene expression and replication in human cells. In an in vitro study with SARS-CoV viruses, siRNA targeted the sequence involved in initiating transcription in coronaviruses, and by silencing gene expression, inhibited the replication of SARS-CoV in Vero E6 cells (30). Currently, there are nine computationally identified conserved regions in the SARS-CoV-2 genome that could be a target of siRNA (30). Nanoparticles help to protect the siRNA from degradation by nucleases, which is a major problem hampering the delivery of bare, unprotected siRNA to target cells (30).

Nanoparticles may also be used for direct pulmonary delivery, allowing for sustained release in the lungs without entering systemic circulation. By modifying the properties of the nanoparticles (particle size, surface area and electrical charge), the optimal distribution of the drug can be achieved (3).

The S-Protein of a SARS-CoV-2 virus that mediates the fusion of the virus particle with the host cell has also become a potential treatment target of fusion-inhibitors (31). In a study 
with MERS-induced infections in the cell culture, the use of nanorods as a delivery material enhanced the inhibitory activity of fusion-inhibitors 10 -fold, revealing great potential for the treatment of SARS-CoV-2 infections (30).

Nanoparticles such as carbon quantum dots (CQD) can reveal antiviral activity by directly inhibiting entry into the host cell (32). The underlying mechanism of action of CQDs is interaction between the functional groups of these nanoparticles and the entry receptors.

An interesting example exhibiting versatility and the broad engineering possibilities of polymeric nanoparticles is their use in generating so-called nanosponges. In nanosponges, nanoparticles are used as a platform coated with cellular membranes of human-derived lung epithelial cells or macrophages, resulting in a display of receptors that SARS-CoV-2 needs for cellular entry (33). To support this, nanosponges were able to neutralize the virus particles and lower viral load in mice.

Finally, nanoparticles stimulate immune system activity. Gold nanoparticles can penetrate various immune cells (e.g. macrophages, dendritic cells, monocytes and lymphocytes) and enhance the production of proinflammatory cytokines, such as interleukin (IL)-1 $\beta$, IL-2, IL-6 and tumor necrosis factor $\alpha$ (34). Importantly, low doses of nanoparticles had an immunostimulatory effect, whereas high doses were proinflammatory or immunotoxic, leading to a drastic decline in the proliferative activity of lymphocytes (34).

Nanomaterials can also be used to reduce the cytokine storm triggered by COVID-19, which can improve the clinical outcomes of patients (35). Graphene nanoplatelets allowed for the absorption and rapid removal of proinflammatory cytokines from human plasma in vitro (36). However, the immunomodulating properties of nanoparticles in COVID-19 require extensive studies, considering the complexity of the immune system and relationships between its individual components. The working principle of nanoparticle-based drugs and delivery systems is presented in Figure 3. 


\section{Use of nanoparticles in specific clinical scenarios}

\section{Reinfection}

Recurrent positive real-time quantitative polymerase chain reaction results for SARS-CoV-2 infection were reported in literature already during the first months of epidemic (37). Consequently, it has become clear that the long-lasting immunity after the natural infection with SARS-CoV-2 is often compromised and consequently does not provide the appropriate protection against reinfection. The underlying pathophysiologic mechanisms are complex and include: (i) the properties of the host immune system and (ii) the virus itself (38).

With regards to the immunity, it has been suggested that the progressive T-lymphocytes exhaustion, observed in the course of COVID-19 (39), expressed by the upregulation of inhibitory markers (e.g. programmed cell death protein 1, also known as PD-1 and T cell immunoglobulin and mucin domain-containing protein 3, also known as TIM3) leads to an impaired T-lymphocytes effector function (40). The resulting ineffective immune response would then contribute to higher risk of reinfection. It has been also suggested that in course of the disease, the massive lysis of the lymphocytes, both through direct viral cytopathogenic effect and through the cytokine storm contributes to this phenomenon (41). Also, migration of numerous cells of immune system to the infection sites, with macrophages, neutrophils and inflammatory cytokines accumulating in the bronchoalveolar lavage fluid has been reported as one of the possible mechanisms (42).

The virus itself exhibits propensity for mutations in its genetic material, causing modifications in epitope structure, which consequently prevents the previously generated antibodies from recognizing the viral antigens (38). In the absence of beneficial activity of antibodies the host does not have the advantage of adaptive immune response and is more susceptible to reinfection (38). 
Nanoparticles may provide a solution to the problem, by exerting a direct immunostimulatory effect. For example, nanoparticles were shown to enhance a humoral response to malaria antigens in mice by interacting directly with the immune system. As already described, nanoparticles have the ability to accumulate in the lymph nodes over a longer period compared with soluble protein immunizations (43). Upon microscopic investigation, the formation of germinal centers was visualized adjacent to the nanoparticles' accumulation sites, indicating that nanoparticles increased antigen delivery by increasing the B-cell response.

The concept of nanoimmunity-by-design assumes that nanoparticles can be tailored to elicit an effective antigen-immune cell reaction and optimal stimulation of immune system, thus allowing for protective, long-lasting immunity (3). Whether this immunostimulatory effect should be applied during the primary infection as an auxiliary therapy option, following the infection as a way of boosting immunity or together with a vaccine as an adjuvant, remains to be investigated.

At the moment, a vaccination is recommended also for patients with prior SARS-CoV2 infection, provided that they have recovered from the acute illness and criteria have been met for the to discontinue isolation (44). Considering that the vaccination programs are already in progress, it is worth considering to administer nanoparticles together with a vaccine as a measure of stimulating the effective immune response. This would be especially advantageous in patients with low antibody levels, or those with evidence of more than 2 infections with SARS-CoV-2, suggesting the insufficient immunization in the course of the disease.

\section{COVID-19 in pregnancy}

Pregnancy constitutes a challenge for the immune system, requiring constant, optimal adaptations in humoral and cellular immune response, both on the local and systemic level (45). In the first trimester, a pro-inflammatory state is beneficial to the embryo in the process of implantation and placentation. This changes in the second trimester, when the anti- 
inflammatory state helps the embryo to grow. Finally, in the third trimester, the proinflammatory stare prepares the body for the initiation of the parturition (46).

In a retrospective cohort study, increased rates of preterm birth, low birth weight, neonatal asphyxia, premature rupture of membrane, and cesarean section delivery were reported in women with confirmed COVID-19 diagnosis during pregnancy, compared to those not infected with SARS-CoV-2 (47). Also, an association of pregnancy with the higher severity of the disease was observed (48). Thus, the need for an effective treatment of COVID-19 in pregnant women is urgent.

One of the mechanisms contributing to worse outcomes in pregnant women is the cytokine storm, promoted by a general proinflammatory state in the $1^{\text {st }}$ and $3^{\text {rd }}$ trimester of pregnancy. In the course of cytokine storm, plasma concentrations of: of interleukins 2 (IL-2), IL-7, IL-10, granulocyte-colony stimulating factor, interferon- $\gamma$-inducible protein 10 , monocyte chemoattractant protein 1, macrophage inflammatory protein 1 alpha, and tumor necrosis factor $\alpha$ are elevated, which aggravates the course of the disease $(46,49)$. Cytokine storm is among the most significant causes of acute respiratory distress syndrome and multiple-organ failure.

Considering the susceptibility of pregnant women for the hyperinflammatory reactions, the immunomodulating properties of nanoparticles seem especially promising as an auxiliary treatment method in pregnant women with COVID-19. There are multiple possibilities of applying nanoparticles and tailoring them for an optimal immunomodulatory effect by adjusting their physicochemical properties, as previously described $(36,50)$. However, there are no evidence-based data regarding immunomodulating properties of nanoparticles in pregnant women. As nanoparticles were previously proven to both stimulate and suppress the immune response, depending on multiple factors (physicochemical and immune properties, route of administration), further studies, preferably on an experimental model, are required to extend 
our understanding of the complex immunological reactions and allow for the controlled management of the immune response.

Also, ongoing discussions regarding safety of medication against COVID-19 for pregnant women and newborns have led to exclusion of pregnant women from clinical trials. This is now being regarded as a major problem in long-term COVID-19 treatment strategy, as it hampers the introduction of potentially effective drugs in this patient's group. Data from international trial registries indicate that less than $2 \%$ of all COVID-19 registered trials include pregnant women (51). Recently published appeal for the inclusion of women in clinical trials (52), together with requests from Coalition to Advance Maternal Therapeutics to the National Institutes of Health (NIH) and to US Food and Drug Administration in this regard (53) show the urgent need for reconsidering the research on therapeutic agents in pregnant women. On the other hand, treatment of COVID-19 is known to induce a number of adverse effects. Most of the studies investigating the treatment options for COVID-19 reported infrequent and mild adverse reactions (54). However, many of them did not provide any data in this regard. Studies conducted on pregnant women have also shown a low rate of side effects and adverse pregnancy outcomes (55). However, low number of trials conducted in the group of pregnant women and methodological shortcomings (lack of control group, low sample sizes, data derived from case reports/case series) do not allow to draw clear conclusions. Therefore, nanoparticles-based delivery systems could be considered not only for the currently approved treatment regimens, but also for the safe introduction of therapeutics during clinical trials. Nanoparticles allow for the deposition of a therapeutic agent to the target site, thus improving its biodistribution and limiting the exposure of remaining tissue to the substance (30). Nanoparticles-based therapies could be the solution to the problem of poor treatment options for pregnant women and allow for the increased safety profile guaranteeing the well-being of the child.

\section{The problem of coinfection.}


Recently the problem of coinfection in the course of COVID-19 has been increasingly recognized. This is probably most accentuated in the case of simultaneous infection with SARSCoV-2 and dengue virus (DENV) (56). However, this problem has also been reported in case of coinfection with influenza virus (57), Streptococcus pneumoniae (58), as well as bacteria or fungi (59). One study found a higher rate of coinfection with influenza virus during a screening procedure, suggesting that it remains often undiagnosed and underrecognized in a clinical setting (59).

The problem of SARS-CoV-2 and DENV coinfection is urgent due to (i) high prevalence of the disease and uncertain rates of coinfection, (ii) similar symptoms and pathophysiological mechanisms of both diseases, (iii) different prognosis in case of coinfection compared to mono-infection with SARS-CoV-2, (iv) occurrence of the disease in the countries with a low socioeconomic status, (v) need for specific containment measures in case of coinfection as opposed to the infection with DENV only.

There has been an 8-fold increase in the number of DENV cases over the last two decades, from 505,430 cases in 2000, to over 2.4 million in 2010, and 5.2 million in 2019 (60). Only in the last years, the reporting of DENV infections have improved globally, which makes the actual occurrence of dengue and the real burden of the disease uncertain. Also, there are continuous outbreaks of dengue in both the Americas, Africa, the Middle East, Asia, and the Pacific Islands (61). The outbreaks make this disease difficult to control, as they exhaust the sanitary capacities and put strain on health systems. In 2020, a great number of dengue hotspots were reported in many Asian and African countries, as well as in continuously affected Brazil (62).

The first case report of a human coinfection of SARS-CoV-2 and DENV, in which both viruses were detected by RT-qPCR at the same time was published in August 2020. It is, at the moment, difficult to assess the extent to which the described phenomenon of coinfection is 
present, considering that both diseases share clinical manifestations and can take an asymptomatic to mild-symptomatic form.

While the proper identification of the infectious agent is crucial, the cross-reactivity between the antibodies directed against SARS-CoV-2 and DENV decreases the specificity of antibodies-based diagnostic tests (e.g. rapid serological tests) which may produce false-positive results (63). The solution to this problem would be the application of molecular diagnostic methods as a point-of-care testing. Recently, multiple point-of-care RNA detection technologies based on isothermal amplification methods were developed, including reverse transcription- recombinase polymerase amplification (RT-RPA) and reverse transcriptionloop-mediated isothermal amplification (RT-LAMP) (64). There are also new, promising nanoparticles-based nucleic acid detection technologies, complementary or even alternative to the above-mentioned.

The first commercial sequencer, using nanopore technology was released in 2014 under the commercial name MinION by Oxford Nanopore Technologies (65). The system recognizes DNA bases by detecting change in pattern or magnitude of electrical current during passage of a DNA molecule through the nanopore (66). This system is designed for DNA detection, however the technology of nanopore analysis allows for detection of multiple nucleic acids including RNA (67). Thus, the possible application for SARS-CoV-2 detection is very promising, considering the effects obtained for MinION system. Advantages of MinION system include its small size, portability and low equipment. The devices is only 10x3x2 $\mathrm{cm}$ large and allows for sample processing using Windows software, in this way fitting into real-life conditions of airports, hospitals and other public facilities, where the operative and rapid diagnostic methods are essential. However, the sequencing capacity of MinION requires improvement with accuracy ranging from $65 \%$ to $88 \%(66,68)$. This result does not show any improvement compared with existing sequencing technologies at the moment and hampers the 
entry of MinION into clinical practice. However, the concept is worth considering and optimizing, as it is does not depend on laboratory infrastructure and thus may be of use in resource-limited scenarios.

\section{Conclusions}

The COVID-19 outbreak has put global pressure on modern societies and healthcare. Nanoparticles offer new prospects for COVID-19 prevention, detection and treatment methods, including vaccine development, nanoparticle-based diagnostic tests and several treatment methods, based on direct antiviral activity of nanoparticles, delivery of therapeutic agents and stimulation of both immunomodulant and immunosuppressive functions. Here, we summarized a broad spectrum of nanoparticle applications in COVID-19 from a clinical perspective with the goal of providing educational value for clinicians interested in the development and/or application of nanoparticle-based technologies and guiding the reader through the complex field of nanotechnology toward prospective, patient-oriented clinical trials as well as applications of nanoparticle-based technologies in the clinical scenarios.

\section{Figure legends:}

Figure 1. The principle of an mRNA-based vaccine delivered by lipid nanoparticles. Lipid nanoparticles protect mRNA from enzymatic degradation. MRNA vaccines transfect myocytes or antigen-presenting cells (APC). The transfected cells (e.g. myocytes) express the mRNAencoded fragments of antigen on the major histocompatibility complex (MHC) class I. In addition, they excrete virus proteins which are recognized by antigen-presenting cells (APC) and expressed on the MHC class II molecules. Both pathways evoke an immunological response against the antigen. Figure created with BioRender.com, licensed version. 
Figure 2. The principle of a gold nanoparticle-based diagnostic test to detect infection with SARS-CoV-2. The sample containing N-protein is deposited in the sample pad and flows across the strip. In the conjugation pad, the N-protein binds to the anti-N-protein antibody labeled with a gold nanoparticle. As the sample passes the test line, the conjugate binds to the immobilized anti-N-protein antibody. At the control line, the conjugate of rabbit IgG antibody labeled with a gold nanoparticle is immobilized by the capture antibodies. The results are visualized as red strips in the test and control lines. Figure created with BioRender.com, licensed version.

Figure 3. The working principle of nanoparticle-based drugs and delivery systems. Sunitinib and erlotinib act as AP2-associated protein kinase 1 (AAK1) inhibitors, consequently blocking viral entry. Their toxicity could be avoided by coupling the AAK1 inhibitors with nanoparticles. Small interfering RNA silences the viral mRNA and thus blocks its gene expression and replication in human cells. Nanoparticles help to protect the siRNA from degradation by nucleases. Nanoparticles may also be used for direct pulmonary delivery, allowing for sustained release in the lungs without entering systemic circulation. Finally, nanoparticles stimulate immune system activity and reduce the cytokine storm. Figure created with BioRender.com, licensed version. 


\section{References:}

1. Lv Y, Ma C, Li X, Wu M. Big data driven COVID-19 pandemic crisis management: potential approach for global health. Arch Med Sci. 2021;17(3):829-37.

2. Kostarelos K. Nanoscale nights of COVID-19. Nature Nanotechnology. 2020;15(5):343-4.

3. Singh $P$, Singh $D$, Sa $P$, Mohapatra $P$, Khuntia A, S KS. Insights from nanotechnology in COVID19: prevention, detection, therapy and immunomodulation. Nanomedicine (Lond). 2021.

4. Cardoso VMdO, Moreira BJ, Comparetti EJ, Sampaio I, Ferreira LMB, Lins PMP, et al. Is Nanotechnology Helping in the Fight Against COVID-19? Frontiers in Nanotechnology. 2020;2(4).

5. Al-Ahmady ZS, Ali-Boucetta H. Nanomedicine \& Nanotoxicology Future Could Be Reshaped Post-COVID-19 Pandemic. Frontiers in Nanotechnology. 2020;2(19).

6. Shin MD, Shukla S, Chung YH, Beiss V, Chan SK, Ortega-Rivera OA, et al. COVID-19 vaccine development and a potential nanomaterial path forward. Nat Nanotechnol. 2020;15(8):646-55.

7. Wankar JN, Chaturvedi VK, Bohara C, Singh MP, Bohara RA. Role of Nanomedicine in Management and Prevention of COVID-19. Frontiers in Nanotechnology. 2020;2(8).

8. Talebian S, Wallace GG, Schroeder A, Stellacci F, Conde J. Nanotechnology-based disinfectants and sensors for SARS-CoV-2. Nat Nanotechnol. 2020;15(8):618-21.

9. Thanh Le T, Andreadakis Z, Kumar A, Gómez Román R, Tollefsen S, Saville M, et al. The COVID19 vaccine development landscape. Nat Rev Drug Discov. 2020;19(5):305-6.

10. Du L, He Y, Zhou Y, Liu S, Zheng BJ, Jiang S. The spike protein of SARS-CoV--a target for vaccine and therapeutic development. Nat Rev Microbiol. 2009;7(3):226-36.

11. Topol EJ. Messenger RNA vaccines against SARS-CoV-2. Cell. 2021;184(6):1401.

12. Baum C, Kustikova O, Modlich U, Li Z, Fehse B. Mutagenesis and oncogenesis by chromosomal insertion of gene transfer vectors. Hum Gene Ther. 2006;17(3):253-63.

13. Reichmuth AM, Oberli MA, Jaklenec $A$, Langer $R$, Blankschtein D. mRNA vaccine delivery using lipid nanoparticles. Ther Deliv. 2016;7(5):319-34.

14. Bessis N, GarciaCozar FJ, Boissier MC. Immune responses to gene therapy vectors: influence on vector function and effector mechanisms. Gene Ther. 2004;11 Suppl 1:S10-7.

15. Alpar HO, Somavarapu S, Atuah KN, Bramwell VW. Biodegradable mucoadhesive particulates for nasal and pulmonary antigen and DNA delivery. Adv Drug Deliv Rev. 2005;57(3):411-30.

16. Foged C. Subunit vaccines of the future: the need for safe, customized and optimized particulate delivery systems. Therapeutic delivery. 2011;2(8):1057-77.

17. Gregory AE, Williamson D, Titball R. Vaccine delivery using nanoparticles. Frontiers in cellular and infection microbiology. 2013;3:13.

18. Luan X, Sansanaphongpricha K, Myers I, Chen H, Yuan H, Sun D. Engineering exosomes as refined biological nanoplatforms for drug delivery. Acta Pharmacol Sin. 2017;38(6):754-63.

19. Qi H, Liu C, Long L, Ren Y, Zhang S, Chang X, et al. Blood Exosomes Endowed with Magnetic and Targeting Properties for Cancer Therapy. ACS Nano. 2016;10(3):3323-33.

20. Pinky, Gupta S, Krishnakumar V, Sharma Y, Dinda AK, Mohanty S. Mesenchymal Stem Cell Derived Exosomes: a Nano Platform for Therapeutics and Drug Delivery in Combating COVID-19. Stem Cell Reviews and Reports. 2021;17(1):33-43.

21. Armstrong JP, Holme MN, Stevens MM. Re-engineering extracellular vesicles as smart nanoscale therapeutics. ACS nano. 2017;11(1):69-83.

22. Seow Y, Wood MJ. Biological Gene Delivery Vehicles: Beyond Viral Vectors. Molecular Therapy. 2009;17(5):767-77.

23. Borges O, Cordeiro-da-Silva A, Tavares J, Santarém N, de Sousa A, Borchard G, et al. Immune response by nasal delivery of hepatitis $B$ surface antigen and codelivery of a $\mathrm{CpG}$ ODN in alginate coated chitosan nanoparticles. Eur J Pharm Biopharm. 2008;69(2):405-16.

24. Zhao K, Chen G, Shi XM, Gao TT, Li W, Zhao Y, et al. Preparation and efficacy of a live newcastle disease virus vaccine encapsulated in chitosan nanoparticles. PLoS One. 2012;7(12):e53314. 
25. Feng $G$, Jiang $Q$, Xia $M$, Lu $Y$, Qiu $W$, Zhao $D$, et al. Enhanced immune response and protective effects of nano-chitosan-based DNA vaccine encoding T cell epitopes of Esat- 6 and FL against Mycobacterium tuberculosis infection. PLoS One. 2013;8(4):e61135.

26. Lee S, Cho AR, Park D, Kim JK, Han KS, Yoon IJ, et al. Reusable Polybenzimidazole Nanofiber Membrane Filter for Highly Breathable PM(2.5) Dust Proof Mask. ACS Appl Mater Interfaces. 2019;11(3):2750-7.

27. . !!! INVALID CITATION !!! .

28. Pérez-López B, Mir M. Commercialized diagnostic technologies to combat SARS-CoV2: Advantages and disadvantages. Talanta. 2021;225:121898.

29. Anselmo AC, Mitragotri S. Nanoparticles in the clinic: An update. Bioeng Transl Med. 2019;4(3):e10143.

30. Itani R, Tobaiqy M, Al Faraj A. Optimizing use of theranostic nanoparticles as a life-saving strategy for treating COVID-19 patients. Theranostics. 2020;10(13):5932-42.

31. Xia S, Liu M, Wang C, Xu W, Lan Q, Feng S, et al. Inhibition of SARS-CoV-2 (previously 2019$\mathrm{nCoV}$ ) infection by a highly potent pan-coronavirus fusion inhibitor targeting its spike protein that harbors a high capacity to mediate membrane fusion. Cell Res. 2020;30(4):343-55.

32. Łoczechin A, Séron K, Barras A, Giovanelli E, Belouzard S, Chen YT, et al. Functional Carbon Quantum Dots as Medical Countermeasures to Human Coronavirus. ACS Appl Mater Interfaces. 2019;11(46):42964-74.

33. Zhang $\mathrm{Q}$, Honko A, Zhou J, Gong H, Downs SN, Vasquez JH, et al. Cellular Nanosponges Inhibit SARS-CoV-2 Infectivity. Nano Lett. 2020;20(7):5570-4.

34. Dykman LA, Khlebtsov NG. Immunological properties of gold nanoparticles. Chem Sci. 2017;8(3):1719-35.

35. Ghanei M. It is time to consider an anti-inflammatory therapy based on the pathophysiology of COVID-19 infection during the right time window? Arch Med Sci. 2021;17(2):546-50.

36. Zheng Y, Pescatore N, Gogotsi Y, Dyatkin B, Ingavle G, Mochalin V, et al. Rapid Adsorption of Proinflammatory Cytokines by Graphene Nanoplatelets and Their Composites for Extracorporeal Detoxification. Journal of Nanomaterials. 2018;2018:6274072.

37. Gousseff M, Penot P, Gallay L, Batisse D, Benech N, Bouiller K, et al. Clinical recurrences of COVID-19 symptoms after recovery: Viral relapse, reinfection or inflammatory rebound? J Infect. 2020;81(5):816-46.

38. Nainu F, Abidin RS, Bahar MA, Frediansyah A, Emran TB, Rabaan AA, et al. SARS-CoV-2 reinfection and implications for vaccine development. Human Vaccines \& Immunotherapeutics. 2020;16(12):3061-73.

39. Diao B, Wang C, Tan Y, Chen X, Liu Y, Ning L, et al. Reduction and Functional Exhaustion of T Cells in Patients With Coronavirus Disease 2019 (COVID-19). Front Immunol. 2020;11:827.

40. Wherry EJ. T cell exhaustion. Nat Immunol. 2011;12(6):492-9.

41. Vabret N, Britton GJ, Gruber C, Hegde S, Kim J, Kuksin M, et al. Immunology of COVID-19: Current State of the Science. Immunity. 2020;52(6):910-41.

42. Liao M, Liu Y, Yuan J, Wen Y, Xu G, Zhao J, et al. Single-cell landscape of bronchoalveolar immune cells in patients with COVID-19. Nat Med. 2020;26(6):842-4.

43. Moon JJ, Suh H, Li AV, Ockenhouse CF, Yadava A, Irvine DJ. Enhancing humoral responses to a malaria antigen with nanoparticle vaccines that expand Th cells and promote germinal center induction. Proc Natl Acad Sci U S A. 2012;109(4):1080-5.

44. Summary Document for Interim Clinical Considerations. https://www.cdc.gov/vaccines/covid19/clinical-considerations/covid-19-vaccines-us.html. Accessed on 08.08.2021

45. Kumar R, Yeni CM, Utami NA, Masand R, Asrani RK, Patel SK, et al. SARS-CoV-2 infection during pregnancy and pregnancy-related conditions: Concerns, challenges, management and mitigation strategies-a narrative review. Journal of Infection and Public Health. 2021;14(7):863-75.

46. Liu H, Wang LL, Zhao SJ, Kwak-Kim J, Mor G, Liao AH. Why are pregnant women susceptible to COVID-19? An immunological viewpoint. J Reprod Immunol. 2020;139:103122. 
47. Yang R, Mei H, Zheng T, Fu Q, Zhang Y, Buka S, et al. Pregnant women with COVID-19 and risk of adverse birth outcomes and maternal-fetal vertical transmission: a population-based cohort study in Wuhan, China. BMC Medicine. 2020;18(1):330.

48. Ellington S, Strid P, Tong VT, Woodworth K, Galang RR, Zambrano LD, et al. Characteristics of Women of Reproductive Age with Laboratory-Confirmed SARS-CoV-2 Infection by Pregnancy Status United States, January 22-June 7, 2020. MMWR Morb Mortal Wkly Rep. 2020;69(25):769-75.

49. Huang C, Wang Y, Li X, Ren L, Zhao J, Hu Y, et al. Clinical features of patients infected with 2019 novel coronavirus in Wuhan, China. Lancet. 2020;395(10223):497-506.

50. Gazzi A, Fusco L, Orecchioni M, Ferrari S, Franzoni G, Yan JS, et al. Graphene, other carbon nanomaterials and the immune system: toward nanoimmunity-by-design. Journal of Physics: Materials. 2020;3(3):034009.

51. Smith DD, Pippen JL, Adesomo AA, Rood KM, Landon MB, Costantine MM. Exclusion of Pregnant Women from Clinical Trials during the Coronavirus Disease 2019 Pandemic: A Review of International Registries. Am J Perinatol. 2020;37(8):792-9.

52. Whitehead CL, Walker SP. Consider pregnancy in COVID-19 therapeutic drug and vaccine trials. Lancet. 2020;395(10237):e92.

53. Coalition to Advance Maternal Therapeutics COVID-19 and pregnant women and lactating women. March 18, 2020. https://s3.amazonaws.com/cdn.smfm.org/media/2268/Final_CAMT_COVID_Letter_March_2020.pdf . Accessed on 09.08.2021

54. Bartoli A, Gabrielli F, Alicandro T, Nascimbeni F, Andreone P. COVID-19 treatment options: a difficult journey between failed attempts and experimental drugs. Intern Emerg Med. 2021;16(2):281308.

55. https://www.medicinesinpregnancy.org/bumps/monographs/MEDICATIONS-USED-TOTREAT-COVID-19-IN-PREGNANCY/ accessed on 02.08.2021

56. Harapan H, Ryan M, Yohan B, Abidin RS, Nainu F, Rakib A, et al. Covid-19 and dengue: Double punches for dengue-endemic countries in Asia. Rev Med Virol. 2021;31(2):e2161.

57. Ozaras R, Cirpin R, Duran A, Duman H, Arslan O, Bakcan Y, et al. Influenza and COVID-19 coinfection: Report of six cases and review of the literature. J Med Virol. 2020;92(11):2657-65.

58. Toombs JM, Van den Abbeele K, Democratis J, Mandal AKJ, Missouris CG. Pneumococcal coinfection in COVID-19 patients. J Med Virol. 2021;93(1):177-9.

59. Rawson TM, Moore LSP, Zhu N, Ranganathan N, Skolimowska K, Gilchrist M, et al. Bacterial and Fungal Coinfection in Individuals With Coronavirus: A Rapid Review To Support COVID-19 Antimicrobial Prescribing. Clin Infect Dis. 2020;71(9):2459-68.

60. Dengue and severe dengue. https://www.who.int/news-room/fact-sheets/detail/dengueand-severe-dengue. Accessed on 6.08.2021

61. https://www.cdc.gov/dengue/areaswithrisk/around-the-world.html. Accesed on 08.08.2021

62. https://www.who.int/news-room/fact-sheets/detail/dengue-and-severe-dengue. Accesed on 08.08.2021

63. Yan G, Lee CK, Lam LTM, Yan B, Chua YX, Lim AYN, et al. Covert COVID-19 and false-positive dengue serology in Singapore. Lancet Infect Dis. 2020;20(5):536.

64. Abdelhamid HN, Badr G. Nanobiotechnology as a platform for the diagnosis of COVID-19: a review. Nanotechnology for Environmental Engineering. 2021;6(1):19.

65. https://nanoporetech.com/products/minion. Accessed on 08.08.2021

66. Lu H, Giordano F, Ning Z. Oxford Nanopore MinION sequencing and genome assembly. Genomics, proteomics \& bioinformatics. 2016;14(5):265-79.

67. Venkatesan BM, Bashir R. Nanopore sensors for nucleic acid analysis. Nature nanotechnology. 2011;6(10):615-24.

68. Laver T, Harrison J, O'neill P, Moore K, Farbos A, Paszkiewicz K, et al. Assessing the performance of the oxford nanopore technologies minion. Biomolecular detection and quantification. 2015;3:1-8. 
The mechanism of action of mRNA-based vaccines utilizing a nanoparticle platform

$8 \begin{gathered}\text { CoV spike } \\ \text { protein }\end{gathered}$

Myocyte vaccine pathway

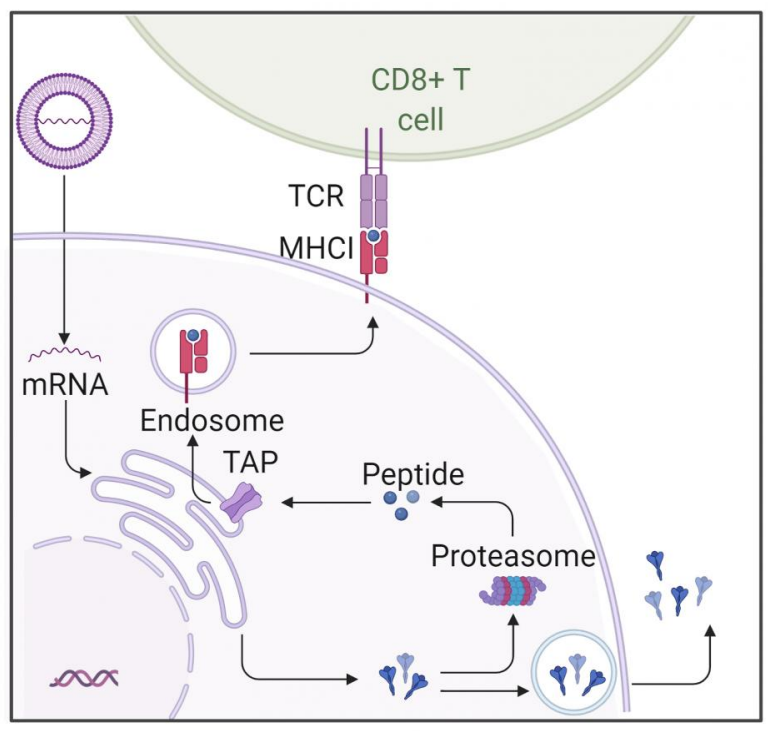

(1) Lipid nanoparticle

APC vaccine pathway

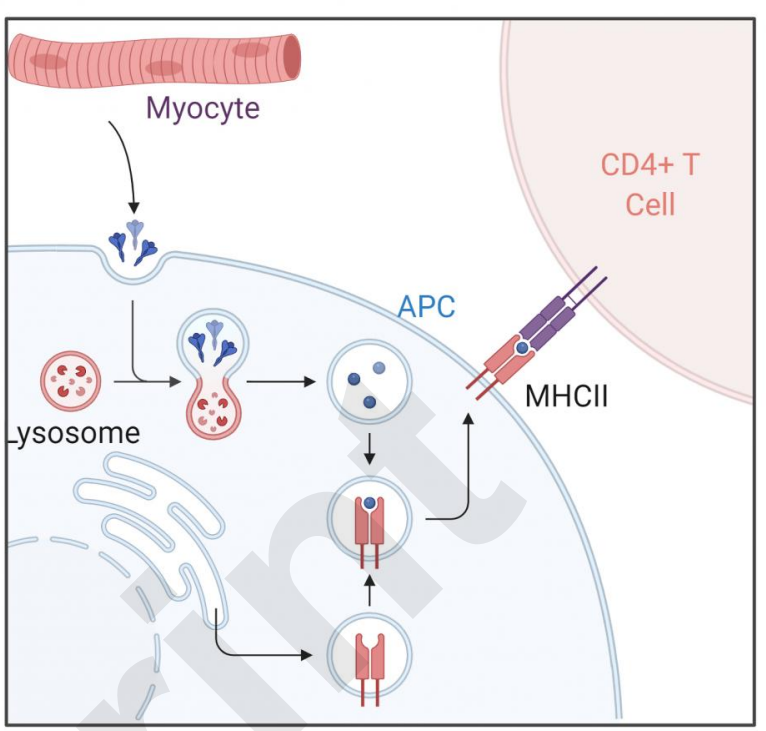


Structure of a lateral flow immunchromatographic assay

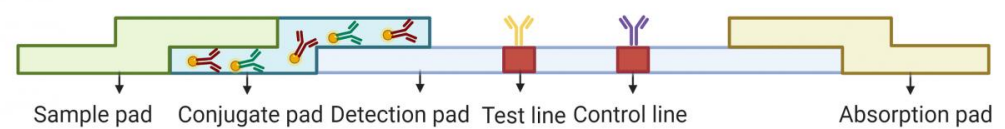

Diagnostic Steps

(1) Sample loading
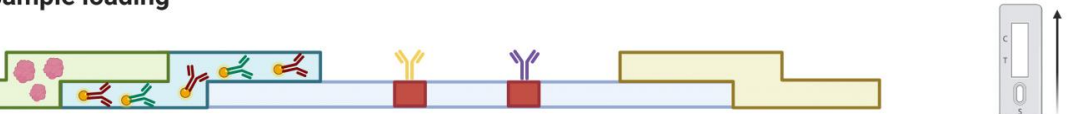

(2) Antibody-antigen recognition
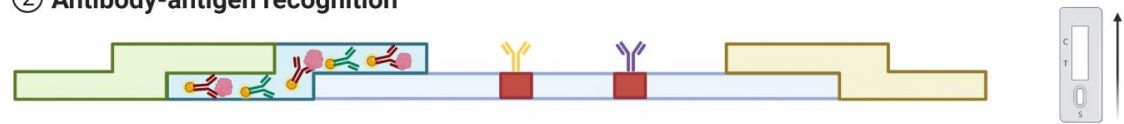

(3) COVID-19 antibody detection

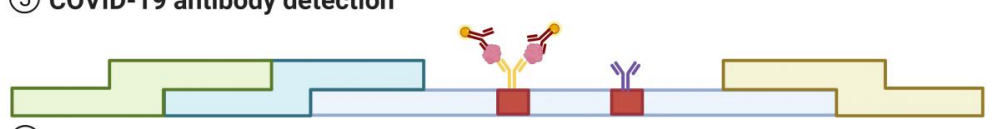

(4) Control antibody detection

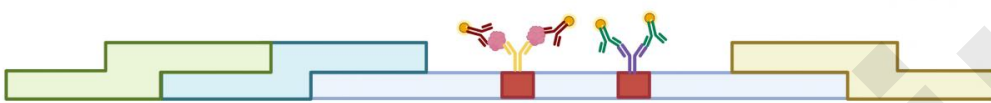

y Anti-N-protein antibody labeled with gold nanoparticle

Rabbit IgG antibody labeled

with gold nanoparticle

Anti-N-protein antibody

Y' Rabbit IgG antibody

N-protein
Interpreting results

Positive Negative Invalid

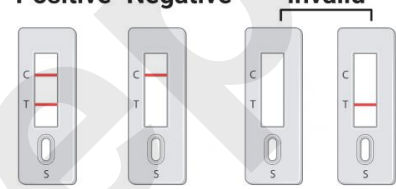




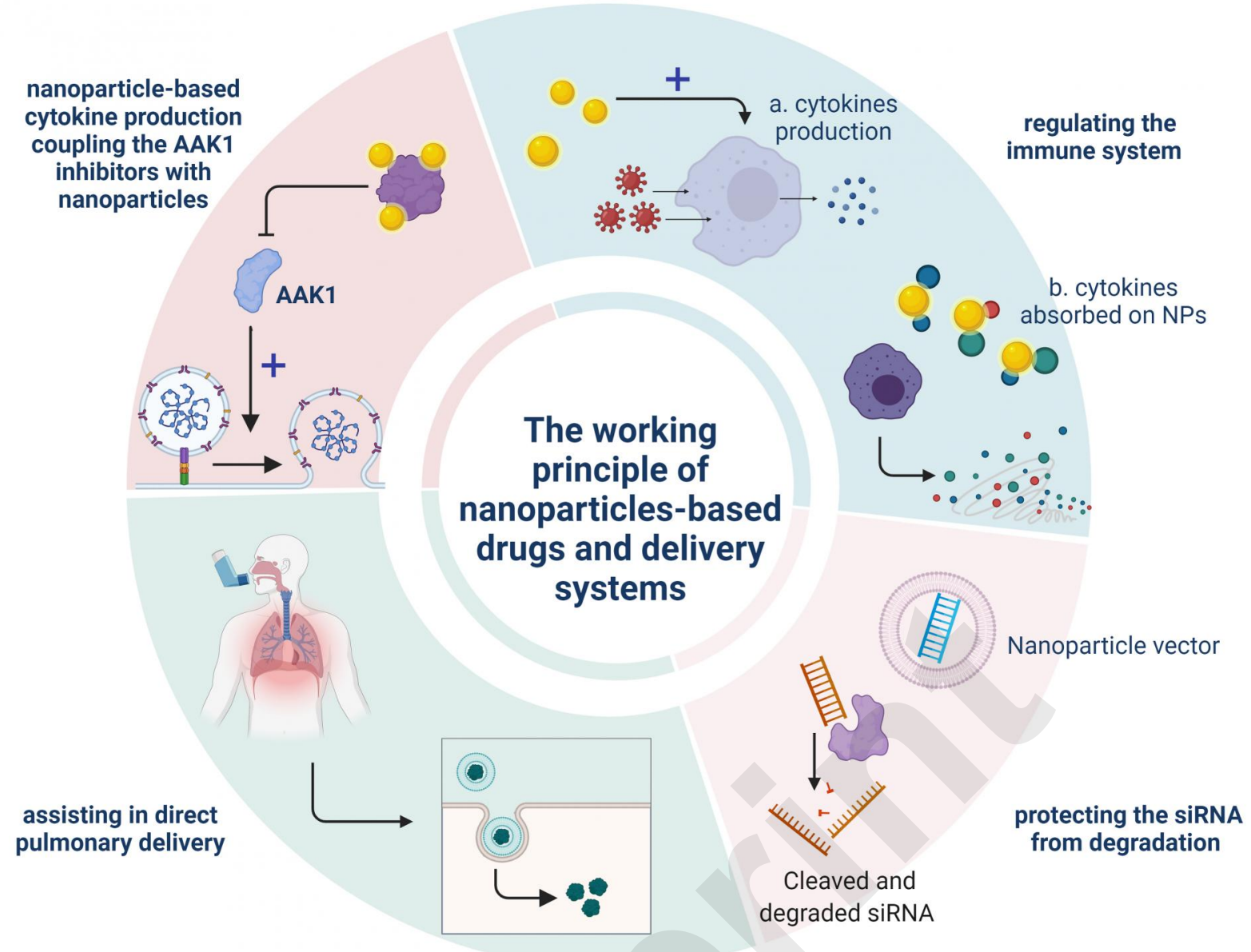

